\title{
La première expérience poétique de Lodovico Dolce. Un exemple de réécriture pétrarquiste
}

\section{Donatella Donzelli}

\section{OpenEdition}

\section{Journals}

Édition électronique

URL : http://journals.openedition.org/cei/616

DOI : $10.4000 /$ cei.616

ISSN : 2260-779X

\section{Éditeur}

UGA Éditions/Université Grenoble Alpes

\section{Édition imprimée}

Date de publication : 15 avril 2006

Pagination : 51-72

ISBN : 978-2-84310-081-9

ISSN : 1770-9571

\section{Référence électronique}

Donatella Donzelli, «La première expérience poétique de Lodovico Dolce. Un exemple de réécriture pétrarquiste », Cahiers d'études italiennes [En ligne], 4 | 2006, mis en ligne le 15 octobre 2007, consulté le 20 avril 2019. URL : http://journals.openedition.org/cei/616 ; DOI : 10.4000/cei.616 


\title{
LA PREMIÈRE EXPÉRIENCE POÉTIQUE DE LODOVICO DOLCE
}

\author{
UN EXEMPLE DE RÉÉCRITURE PÉTRARQUISTE
}

\author{
Donatella Donzelli \\ Université de la Sorbonne Nouvelle-Paris 3
}

Le début de la longue carrière de l'auteur-éditeur vénitien Lodovico Dolce, mort en 1568, remonte à 1532, année de la publication d'un ouvrage en tercets intitulé $\mathrm{Il}$ sogno di Parnaso ${ }^{1}$. Ce petit poème de 463 vers, qui n'a jamais fait l'objet d'une étude approfondie ${ }^{2}$, n'est pas d'une grande originalité, mais peut nous intéresser dans la mesure où il permet d'analyser le travail d'un débutant qui s'initie à la carrière poétique et qui décide de traduire en vers l'expérience de cette initiation. L'escalade du Parnasse jusqu'à l'arrivée au siège d'Apollon et des Muses, qui y est racontée, est en effet une métaphore de l'accès à la gloire poétique. Ce texte, dont le but était, à notre sens, de présenter l'auteur à l'Italie lettrée, est analysable comme une sorte d'exercice rhétorique. Il fallait que Dolce montre à la fois ses connaissances littéraires, sa capacité de faire un travail équilibré entre création et mémoire, et son habileté à faire de son ouvrage

1. L'édition, dont le titre complet est Il sogno di Parnaso con alcune altre rime d'amore, est dépourvue de mention du lieu d'impression, du nom du typographe et de la date. Toutefois, cette dernière est suggérée par la lettre dédicatoire de Lodovico Dolce Al reveren. Monsignore Messer Bendetto Bernardo Prothonotario Apostolico qui porte la mention suivante: «In Vinegia. Il primo di Maggio. M.D.XXXII.». Il n'existe aucune édition moderne de cet ouvrage dont il reste très peu d'exemplaires: celui de la Bibliothèque Nationale Marciana de Venise, que nous avons consulté, celui de la Bibliothèque des Lincei de Rome, celui de la Bibliothèque municipale de Pérouse et celui de la British Library.

2. On peut trouver quelques observations critiques sur $I l$ sogno di Parnaso chez Luigi FIRPO, "Allegoria e satira in Parnaso", Belfagor, I (1946), p. 673-699; Marc Fumaroli, Académie, Arcadie, Parnasse: trois lieux allégoriques du loisir lettré, in IDEM, L'école du silence. Le sentiment des images au XVII siècle, Paris, Flammarion, 1994, p. 19-36; et Christopher CAIRNS, The Dream of Parnassus, Aretino's Heritage and Poligrafi, in Pietro Aretino and the Republic of Venice. Researches on Aretino and his Circle in Venice 1527-1556, Florence, Olschki, 1985, p. 231-249. 
un réservoir de topoi, déclarant par la même occasion son adhésion à un courant poétique. Il sogno di Parnaso remplit toutes ces conditions. Dolce inscrit en effet son poème dans la tradition, longue et fortunée, de la vision-triomphe, inaugurée par Dante et fixée en tant que genre littéraire par Pétrarque et Boccace. Loin de se borner à l'imitation de ses modèles, il essaye également d'innover en introduisant et développant le motif du Parnasse, siège de la gloire poétique.

Parmi les modèles de vision-triomphe que Lodovico Dolce avait à sa disposition ${ }^{3}$, la Comédie et les Triomphes représentent certainement, pour ce qui est de la structure, les deux sources d'inspiration principales du Sogno di Parnaso. Les deux ouvrages fournissent en effet au petit poème de Dolce son canevas. Toute la partie du Sogno di Parnaso consacrée à la description de la phase initiale du rêve de Lodovico est un enchaînement d'échos de la Comédie. Ce n'est qu'au moment où commence le long défilé des personnages célèbres que le poème de Dante, sans pour autant s'éclipser complètement, cède la place aux Triomphes de Pétrarque ${ }^{4}$.

Un bref résumé du poemetto, qui a pour protagoniste l'auteur luimême ${ }^{5}$, peut nous fournir un premier aperçu de la façon dont Dolce s'est servi de ces deux sources. Le poète prétend raconter un rêve qu'il aurait fait un jour de printemps au petit matin (v. 1-9). Le premier élément qui se présente à lui au moment où il tombe dans les bras de Morphée, est un bois traversé de plusieurs chemins (v. 10-12). Envahi par un sentiment de gaieté, il parcourt ce lieu jusqu'à aboutir au pied d'une très haute montagne à l'aspect effrayant (v. 13-21). Lodovico commence à la gravir, malgré la présence de pierres et d'épines qui rendent difficile la tâche et ralentissent sa marche en le décourageant à poursuivre sa route (v. 22-27). Afin de l'aider à ne pas céder à la fatigue et à gravir cette montagne en vue de la découvrir, se présente à lui un guide qui l'accompagnera tout au long de son voyage. Il s'agit d'une femme sans nom à l'apparence presque divine qui surgit à l'improviste (v.29-33). Elle a pour mission de lui dévoiler le nom de la montagne (v. 34-51), de lui expliquer quelles sont les personnes destinées à ce lieu (v. 52-60), de l'inciter dans un premier temps à s'enga-

3. Pour une analyse de bon nombre de ces exemples nous renvoyons à Luigi FIRPO, Allegoria e satira in Parnaso..., cit.; Francesco GANDOLFo, Il «dolce tempo». Mistica, ermetismo e sogno nel Cinquecento, Rome, Bulzoni, 1978; Claudia PeIrone, Storia e tradizione della terza rima. Poesia e cultura nella Firenze del Quattrocento, Turin, Tirrenia, 1990.

4. Des échos de la Comédie reviennent notamment dans les passages où le défilé des personnages s'arrête momentanément pour insérer des descriptions de lieux ou introduire un nouveau groupe de personnages.

5. Le récit est raconté à la première personne. 
ger dans l'épreuve de cette escalade en lui assurant son aide et en mettant l'accent sur l'exclusivité de cette expérience qui mérite d'être vécue (v. 6178), et de l'encourager ensuite à atteindre le sommet sans reculer devant les difficultés, passage obligé pour pouvoir accéder à l'édifice qui se trouve au bout du chemin et qui récompense tous les efforts (v.79-111). Elle l'empêche de se contenter de la vue du premier lieu paradisiaque qui se présente à eux après l'étape initiale de cette dure escalade, en le stimulant dans sa volonté d'atteindre le but de ce parcours périlleux, rendu difficile par la présence d'aspérités (v.112-138), et elle lui fait finalement visiter l'édifice aux sept portes qui se trouve sur le sommet de la montagne en lui dévoilant, en outre, l'identité de chaque personnage qui l'occupe (v. 139fin).

Ce résumé laisse déjà entrevoir que toute la partie destinée à décrire le parcours du protagoniste vers le sommet du Parnasse foisonne de renvois à des éléments de la Comédie que Dolce modifie tout en les laissant volontairement identifiables. Ainsi, la selva oscura, symbole de la corruption de l'humanité, subit un renversement de sens et se transforme en un bois apaisant qui représente vraisemblablement la vie de l'auteur avant qu'il ne décide d'entreprendre la difficile ascension vers la célébrité. La montagne du Purgatoire change de nom (Parnasse) et devient un purgatoire pour les écrivains. Ce n'est pas la louve qui fait obstacle au poète mais l'aspérité même du chemin, pour la présence d' "aspre et torte pietre» qui symbolisent les difficultés et entravent le parcours. Le guide, qui rappelle à la fois Virgile, Béatrice et Matelda, a la tâche d'accompagner le poète non pas dans un voyage de purification couronné par la contemplation de Dieu, mais dans un parcours qui mène à l'obtention de la gloire poétique ${ }^{6}$. Et le nobile castello du chant IV de l'Enfer est remplacé par le siège d'Apollon et des Muses7. La dimension eschatologique, fortement présente dans

6. Pour une analyse du personnage du guide, nous nous permettons de renvoyer à notre article "Il sogno di Parnaso" de Lodovico Dolce (1532), à paraître dans les actes du colloque international Permanence et changement dans la culture italienne des années Trente du XVI siècle (Paris, 3-5 juin 2004).

7. D’ailleurs, dans l'édifice imaginé par Dolce on entre par sept portes ("Qui, fatto non cred'io da mortal mano, / Surgeva un Edificio di tal sorte, / Che qui non cape in intelletto humano. / "A questo entrar si può per sette porte, / — Disse la Guida - ma vi giungon rari” " (Il sogno di Parnaso, v. 142-146), exactement comme dans le nobile castello de Dante: "Venimmo al piè d'un nobile castello, / sette volte cerchiato d'alte mura, / difeso intorno d'un bel fiumicello. // Questo passammo come terra dura; / per sette porte intrai con questi savi: // giugnemmo in prato di fresca verdura» (Inferno IV, 106-111). Toutes nos citations de la Comédie d'après l'édition par les soins de Natalino Sapegno, Florence, La Nuova Italia, 1981-1983, 3 vol. 
l'ouvrage dantesque, est donc évincée en faveur d'un retour au mythe païen du Parnasse dans le cadre d'une initiation à la vie lettrée ${ }^{8}$. Or, comme dans le nobile castello, dans la demeure d'Apollon et des Muses on rencontre des personnages illustres; mais le nombre considérable de ces personnages et leur disposition sont suggérés par les Triomphes de Pétrarque dont Dolce propose une véritable réécriture, la seule partie originale étant celle qui concerne les personnages de son époque. De cette manière, le poète rend hommage autant au fondateur de la visiontriomphe (Dante) qu'à celui qui en a fait définitivement un genre littéraire (Pétrarque).

Le présent article étant consacré au pétrarquisme de Dolce, nous nous bornons à ces quelques remarques sur les éléments dantesques du Sogno di Parnaso. Nous focaliserons en revanche notre attention sur les échos de Pétrarque et de ses imitateurs. Nous analyserons d'abord, à travers quelques exemples, la réécriture des Triomphes dans la présentation des personnages célèbres qui occupe la deuxième partie du Sogno di Parnaso. Ensuite nous nous attarderons sur la façon dont Dolce combine, dans l'ensemble de son poème, l'imitation de Pétrarque avec l'imitation d'autres auteurs de la tradition italienne, notamment des pétrarquistes.

Dans la partie du Sogno di Parnaso consacrée aux personnages domine le même goût pour l'énumération érudite qui est à la base des Triomphes. La quasi-totalité des personnages cités provient du poème de Pétrarque 9 . Mais l'imitation des Triomphes ne se limite pas à la reprise d'une série de noms. Elle touche également à la structure donnée au défilé. En s'inspirant notamment du Triumphus Fame ${ }^{10}$, Dolce répartit ses personnages en quatre groupes formés respectivement par les philosophes de l'Antiquité,

8. Le parcours proposé par Dolce ressemble à première vue à un voyage initiatique, mais il n'est rien d'autre qu'une métaphore du parcours du poète-écrivain qui entreprend la carrière littéraire en se consacrant notamment au plus noble parmi les arts, la poésie, dans l'espoir de devenir un jour célèbre.

9. Les seules exceptions sont le poète Gallus et les philosophes Ptolémée, Théopompe, Archytas, Thalès, Gorgias et Xénophane. Sur ces six philosophes, deux sont tirés de la Comédie. Le nom du poète Gallus ("Gallo, che amando altrui se stesso offese», v. 410) est en revanche suggéré par Bembo: "Gallo che se stesso offese» (Stanze [...] recitate per giuoco [...] la sera del Carnassale MDVII, octave 21, in Pietro Bembo, Prose e Rime, ed. C. Dionisotti, Turin, UTET, 1966, p. 660).

10. Dans le premier des trois chapitres du Triumphus Fame Pétrarque fait défiler les héros romains, dans le deuxième leurs ennemis et dans le dernier les philosophes et d'autres personnalités du monde latin. 
les érudits et historiens latins, les héros romains et leurs ennemis ${ }^{11}$ et, pour finir, les poètes de l'Antiquité, suivis par une série de personnages célèbres du XIV ${ }^{e}, X^{e}$ et XVI $I^{e}$ siècles. Par rapport à Pétrarque, Dolce a donc inversé l'ordre des trois séries de personnages et a séparé les philosophes des personnalités du monde latin qui, dans les Triomphes, apparaissent en revanche mélangés; en outre il a ajouté le groupe des poètes. En effet, les poètes de l'Antiquité, ainsi que les représentants du stilnovo, sont puisés dans le quatrième chapitre du Triumphus Cupidinis ${ }^{12}$. Dans le but de donner une touche personnelle aux galeries d'hommes célèbres empruntées à Pétrarque, Dolce modifie la disposition des personnages à l'intérieur des différents catalogues. Sur le plan de la représentation des personnages, il reste en revanche plutôt fidèle à son modèle. Soucieux, comme Pétrarque, d'éviter une sèche et aride accumulation de noms, Dolce aspire, généralement, à une représentation des personnages par "médaillons", capable de les immortaliser par l'évocation d'un fait liés à leur nom et de susciter ainsi une émotion chez le lecteur.

Mais l'attachement aux Triomphes de Pétrarque se remarque notamment au niveau des tesselles qui servent à décrire un personnage exemplaire ou une série de personnalités. Dolce fait d'ailleurs preuve d'une certaine passivité à l'égard des solutions proposées dans les Triomphes, au point que le lecteur a souvent l'impression de se trouver face à une réécriture à la limite du calque. Nombreux sont en effet les vers tirés notamment du Triumphus Fame ainsi que du Triumphus Cupidinis (références principales pour tous les auteurs de triomphes) que Dolce a réécrits en se bornant parfois à de légères retouches. Dolce se limite souvent à remplacer simplement un substantif ou un adjectif ou encore un verbe par un synonyme, ou à changer un temps verbal. Ainsi, l'expression "duo chiari troiani ", par laquelle Pétrarque avait désigné Hector et Énée (Triumphus Fame II, 10) est transformée par Dolce in "ambi dui li famosi Troiani» (Il sogno di Parnaso, v. 337) ${ }^{13}$. Toutefois, il arrive également à Dolce de changer la syntaxe d'une phrase tout en conservant certains éléments de celle de Pétrarque. C’est par exemple le cas du tercet de Pétrarque résumant

11. Tout en demeurant distincts, ces deux groupes forment un bloc unique. Dolce a en effet imaginé pour eux une cohabitation dans un château auquel on peut accéder par des portes d'acier et qui se trouve à l'intérieur de l'édifice accueillant l'intégralité des personnages illustres.

12. Les personnages en question sont Orphée, Alcée, Pindare, Anacréon, Virgile, Ovide, Catulle, Properce, Tibulle, Sapho, Dante, Guido et Cino da Pistoia. Voir Petrarca, Triumphus Cupidinis IV, 13-34.

13. Toutes nos citations des Triomphes d'après Francesco Petrarca, Triumphi, a cura di M. Ariani, Milan, Mursia, 1988. 
trois épisodes célèbres de l'Énéide ${ }^{14}$ :

L'altro è colui [Énée] che pianse sotto Antandro

la morte di Creusa, e 'l suo amor [Lavinia] tolse a que' [Turne] che'l suo figliuol [Pallas] tolse ad Evandro.

(Triumphus Cupidinis I, 106-108)

Les vers de Pétrarque sont transformés de la manière suivante:

Quel [Énée] che pianse la moglie sotto Antandro, seco havea quel giovenetto degno [Pallas] di cui fu ciecco et privo Evandro.

(Il sogno di Parnaso, v. 340-342)

Dolce s'est donc limité à une paraphrase du premier vers de Pétrarque où seule la position des mots a changé et le nom de Créuse reste sousentendu. Pour les deux autres vers, en revanche, sa réécriture évince l'élément de la rivalité en amour entre Enée et Turnus pour insister sur la mort injuste et prématurée de Pallas.

Parfois, Dolce adapte, sans presque les modifier, des vers ou des expressions à des personnages autres que ceux à qui ces vers et expressions étaient originellement destinés. Ainsi, le vers que Pétrarque avait consacré au général romain Lucius Papirius Cursor: " $\mathrm{E}$ quanto in arme fu crudo et severo " (Triumphus Fame I, 97), passe, sans grands changements, à Sylla : "Mario è collà con quel [Sylla] ch'a giorni suoi / Tanto ne l'arme fu crudo et severo" (Il sogno di Parnaso, v.310-311). De même, les vers que Pétrarque consacre à Mucius Scaevola: «e chi [Scaevola] a grande opra del nemico stuolo / mosse la mano indarno, e poscia l'arse, / sì seco irato che non sentì il duolo ${ }^{15}$ " sont réorganisés par Lodovico afin de les adapter à deux personnages: Archimède et un philosophe (sans doute Anaxarque). Ce dernier nous est présenté par le recours à la dernière partie du vers de Pétrarque que Dolce reformule à sa convenance: «Vedi quel ch'al morir non sente il duolo» (Il sogno di Parnaso, v. 226). L'expression «nemico stuolo", qui se trouve au début du tercet de Pétrarque, est en revanche insérée dans un nouveau contexte ayant pour protagoniste Archimède: «Et Archimede, che nel patrio nido / rimase estinto dal nemico stuolo» (Il sogno di Parnaso, v. 227-228) ${ }^{16}$.

14. Énée quitte Troie après avoir perdu sa femme Créuse (Énéide II, 735-740 et III, 5-7); une fois arrivé en Italie, Énée épouse Lavinia, la fille du roi du Latium que Turnus avait demandée en mariage (Énéide VII, 55-101); la mort de Pallas, tué par Turnus pour se venger de l'alliance entre Evandre, le père de Pallas, et Énée (Énéide X, 439-489).

15. Triumphus Fame I, 82-84. Dorénavant, tous les mots et les passages en italique devront être considérés comme soulignés par nous.

16. Le tercet consacré à Mucius Scaevola sera par la suite paraphrasé par Dolce pour présenter 
Mais il n'est pas rare non plus que Dolce se sert de l'ensemble des Triomphes ainsi que des Rerum Vulgarium Fragmenta pour construire les vers concernant un personnage. Souvent il reprend des vers presque mot à mot tout en les adaptant à un nouveau contexte. Parfois son intervention se borne à modifier un mot, à changer de temps verbal ou à remplacer un mot par un synonyme. Ainsi, le vers "volando al ciel colla terrena soma" de la célèbre chanson de Pétrarque $O$ aspectata in ciel beata e bella ( $R v f 28$, 78), qui se refere à Giacomo Colonna, est légèrement retouché pour être adapté au personnage de Platon: "Sappi ch'egli è il divino gran Platone / Che volò al ciel con le terrene some" (Il sogno di Parnaso, v. 203-204) ${ }^{17}$.

Les exemples que nous venons de fournir montrent bien que Pétrarque est une présence considérable au sein du Sogno di Parnaso. Mais l'apport ne se limite pas seulement à la partie consacrée au défilé des personnages. Certes, au niveau de la structure, l'ouvrage de Dolce se compose de deux parties dont la première repose clairement sur le souvenir de la Comédie, alors que la seconde se présente presque comme un calque des Triomphes. Toutefois, sur le plan du langage, la séparation entre ces deux sources n'est pas si nette, car elles se superposent et interagissent entre elles. Même la partie qui rappelle de près la Comédie use d'un langage inspiré des Triomphes et des Rerum Vulgarium Fragmenta. Sur les souvenirs en langue vulgaire de Pétrarque viennent en outre se greffer de nombreux autres échos provenant notamment de la Comédie de Dante, de l'Amorosa Visione et des Rime de Boccace, du Morgant de Pulci, des ouvres en vers de Laurent le Magnifique, des Rime de Politien, Correggio, Tebaldeo, Vittoria Colonna et Veronica Gambara ${ }^{18}$, de l'Hypnerotomachia Poliphili

le personnage qui en est le protagoniste et rappeler, comme Pétrarque, l'épisode de 508 av. J.-C. dans lequel ce héros romain laissa brûler sa main droite sur le feu pour se punir d'avoir tué une autre personne à la place du roi étrusque Porsenne qui était en train d'assiéger Rome: «Mutio che, mosso per occider quello / Capo de gli nemici [Porsenne], errando poi / Con la man che fallio si fe'ribello" (Il sogno di Parnaso, v. 307-309).

17. Toutes nos citations des Rerum Vulgarium Fragmenta (dorénavant Rvf) d'après Francesco Petrarca, Canzoniere, ed. G. Contini, Turin, Einaudi, 1964.

18. Les Rime de cet auteur, imprimées dans leur totalité pour la première fois seulement en 1759, mais en partie publiées au cours du Cinquecento dans les recueils des poètes pétrarquistes, pouvaient avoir été portées à la connaissance de Dolce par l'entremise de son ami l'Arétin. Veronica Gambara et ce dernier entretenaient en effet une correspondance. En outre, l'Arétin était aussi ami de Bembo, lequel appréciait cette femme qui avait l'habitude de lui soumettre ses vers. En ce qui concerne les Rime de Vittoria Colonna, elles aussi étaient encore inédites à cette époque. Imprimées pour la première fois à Parme en 1538 (Rime de la divina Vittoria Colonna marchesa di Pescara, In Parma, presso Antonio Viotti), les Rime de Vittoria Colonna devaient déjà circuler sous 
de Francesco Colonna, du Roland Amoureux de Boiardo, de l'Arcadie et des Sonetti e canzoni de Sannazar, des Rime et des Stanze de Bembo, des Rime de l'Arioste ${ }^{19}$ et surtout de son Roland furieux ${ }^{20}$.

L'ouvrage de Dolce s'apparente en effet à un véritable travail de marqueterie littéraire où s'imbriquent des réminiscences de textes de statut et de tonalité très différents. Dolce non seulement montre ainsi qu'il s'est bien approprié toutes les œuvres importantes de la tradition poétique italienne, mais il leur rend aussi hommage, en soldant d'une certaine façon sa dette à l'égard des auteurs qui ne sont pas explicitement cités dans le Panthéon des modernes. Les personnalités de l'Italie lettrée qui peuplent les Saturnia Regna de Dolce sont en effet presque exclusivement d'origine vénitienne, car ce Panthéon se veut un éloge du pétrarquisme et de son principal représentant vénitien, Pietro Bembo, ainsi qu'une célébration de la culture vénitienne et de son marché du livre. Les auteurs toscans, antagonistes principaux de Venise, ne pouvaient donc pas trouver leur place dans ce Parnasse ${ }^{21}$.

En se présentant à l'Italie lettrée, il était important pour Dolce, encore jeune et inconnu, de démontrer son adhésion au pétrarquisme, non seulement en exaltant ses représentants les plus significatifs, mais aussi en manifestant concrètement sa volonté d'écrire à la manière de Pétrarque. En effet, sur le plan linguistique, Il sogno di Parnaso est truffé de réminiscences des Triomphes, des Rerum Vulgarium Fragmenta et des ouvrages des principaux pétrarquistes de l'époque. Dolce fait donc de son ouvrage une sorte d'exercice de rhétorique où l'imitation de la poésie en volgare de Pétrarque domine largement sur les autres sources qui l'ont inspiré. Le poème de Dolce se présente ainsi comme une sorte de collage de réécritures, plus ou moins libres, de vers de Pétrarque laissés volontairement reconnaissables. Pour cette opération de réécriture, Dolce se sert générale-

forme manuscrite dans l'entourage de Bembo et de l'Arétin, deux de ses amis. Selon Tobia Toscano (Appunti sulla tradizione delle Rime amorose di Vittoria Colonna. In margine a una sconosciuta raccolta napoletana di sonetti in morte di Francesco Ferrante d'Avalos, in Tobia R. TOSCANO, Letterati Corti Accademie. La letteratura a Napoli nella prima metà del Cinquecento, Naples, Loffredo Editore, 2000, p. 25-84), les Rime de Vittoria Colonna avaient commencé à être diffusées dans la péninsule italienne à partir de 1530 . Dolce aurait donc pu les connaître grâce à l'Arétin.

19. Même si la publication des Rime de l'Arioste fut posthume, on ne peut pas exclure qu'elles circulaient déjà sous forme manuscrite.

20. Si l'on se base sur la date de la dédicace du Sogno di Parnaso, qui est du $1^{\text {er }}$ mai 1532, il est vraisemblable que, pour son premier ouvrage, qui ne comporte pas de date d'impression, Dolce se soit servi de l'édition du chef-d'œuvre de l'Arioste paru en 1521, la troisième édition étant du $1^{\text {er }}$ octobre 1532.

21. Pour une analyse de ce Panthéon, nous nous permettons de renvoyer à notre étude "Il sogno di Parnaso" de Lodovico Dolce..., cit. 
ment d'autres vers ou expressions de Pétrarque qu'il alterne, de temps en temps, avec des réminiscences d'autres auteurs, de préférence pétrarquistes.

La façon que Lodovico Dolce a de jouer avec ses sources est manifeste dès l'exorde:

Già lieta del suo Vecchio uscia di seno,

Co'bei crin d'oro, la vermiglia Aurora

Facendo in ogni parte il ciel sereno.

Et già con la ruggiada in la fresc'ora

S'udiva Progne et la sorella mesta [Philomèle],

Che de gli antichi guai si lagna et plora.

(Il sogno di Parnaso, v. 1-6)

La structure et les images de cet exorde ont été suggérées par les incipit des Triomphes et du chant neuf du Purgatoire ${ }^{22}$. Mais bien d'autres sources encore ont été mobilisées pour en compléter la rédaction. En proposant sa version du mythe de Tithonos, topos parmi les plus répandus pour se référer à l'aube, Dolce se sert, pour l'image de l'Aurore, autant de la représentation fournie par Pétrarque dans le sonnet Quand'io veggio dal ciel scender l'aurora ("l'aurora / co la fronte di rose et co' crin' d'oro", Rvf 291, 1-2) que de celle qu'on retrouve dans Boccace, Pulci, Tebaldeo et Sannazar où elle est décrite comme "vermiglia ${ }^{23}$. Pour le dernier vers du premier tercet, en revanche, Dolce revient à nouveau à Pétrarque, mais il s'agit cette fois du Pétrarque des Triomphes. Son vers est en effet une sorte de calque de "[lo spirto] fatto avea in quella parte il ciel sereno" du Triumphus Mortis (I, 153). Le passé du verbe a été transformé en un gérondif et l'adjectif quella a été remplacé par ogni. Ensuite, Dolce poursuit avec un autre thème très cher à la littérature et en particulier aux pétrarquistes, celui du début printanier exprimé par le mythe ovidien de Procné et Philomèle. Innombrables sont en effet les exemples de ce mythe en tant qu'élément constitutif du printemps. Parmi les exemples de l'image des deux sœurs saisies en train de pleurer sur leurs malheurs, il semblerait que

22. Pour l'analyse de cet exorde, voir également notre article cité à la note précédente.

23. Voir Giovanni BoCCACCIO, Decamerone III, Introduzione: "L'aurora già vermiglia cominciava, appressandosi il sole, a divenir rancia" (Tutte le opere di Giovanni Boccaccio, ed. Vittore Branca, vol. IV, Milan, Mondadori, 1976) ; Luigi PulcI, Morgante VI, 2: «Era il sol, dico, al balcon d'orïente / e l'Aurora si facea vermiglia» (ed. D. Puccini, Milan, Garzanti, 1989, d'après l'édition De Robertis, Florence, 1962); Antonio Tebaldeo, Rime 148, 2: «Non più al nascer del giorno, come suole, / si mostra la vermiglia e vaga Aurora» (ed. T. Basile et J.J. Marchand, Modène, Panini, 1992, vol. II, 1) ; Jacopo SANNAZARO, Arcadia XI, 14: "E già in questo la vermiglia Aurora alzandosi sovra la terra, significava a' mortali la venuta del sole» (in Opere volgari, ed. A. Mauro, Bari, Laterza, 1961). 
Dolce ait choisi comme modèle l'exorde du Morgant: "Era nel tempo quando Filomena / Con la sorella si lamenta e plora, / Ché si ricorda di sua antica pena" (Morgante I, 3). Le nom de Philomèle est remplacé par celui de Procné, et les deux verbes sont déplacés à la fin de la phrase. Mais on relève dans cette réécriture d'un tercet de Pulci surtout l'utilisation conjointe de Pétrarque et de Sannazar. La périphrase «sorella mesta», qui vient remplacer le laconique "sorella" de Pulci et qui sert à désigner cette fois Philomèle, est en effet un écho de «la mesta Filomena» qui prend place à l'intérieur d'un tableau estival évoqué par Sannazar dans son Arcadia $^{24}$. Ensuite, Dolce se réfere à un autre exemple d'utilisation du mythe ovidien, appliqué cette fois par Sannazar au contexte printanier:

O Filomena, che gli antichi guai

rinovi ogni anno, e con soavi accenti

da selve e da spelunche udir ti fai;

e se tu Progne, è ver c'or ti lamenti

né con forma ti fur tolti i sensi,

ma del tuo fallo ti lagni e penti ${ }^{25}$.

Toutefois Lodovico ne se sert que du premier et du dernier de ces vers. Du premier, il emprunte en effet l'expression "antichi guai», qui prend la place de l'«antica pena» de Pulci. Du dernier, par contre, il récupère l'expression «ti lagni e penti» qui, avec le «si lamenta et plora» du Morgant auquel il vient se mélanger, lui suggère vraisemblablement l'idée de reprendre des Triomphes l'expression «si lagna et plora». Pétrarque l'avait employée dans son Triumphus Cupidinis à propos de Cornelia, la dernière femme de Pompée, afin d'en rappeler la douleur éprouvée pour la perte de son mari et le ressentiment envers Ptolémée qui avait trahi son mari et l'avait fait assassiner ${ }^{26}$ : «Vedi quel grande il quale ogni uomo onora; / egli è Pompeo, et à Cornelia seco, / che del vil Tholomeo si lagna e plora» (Triumphus Cupidinis III, 13-15) ${ }^{27}$.

24. «E per li ombrosi rami le argute cicale cantando si affatigavano sotto al gran caldo; la mesta Filomena da lunge tra folti spineti si lamentava; cantavano le merole, le upupe e le calandre; pingeva la solitaria tortora per le alte ripe; le sollecite api con suave sussurro volavano intorno ai fonti. Ogni cosa redoliva de la fertile estate" (Arcadia X, 59).

25. Arcadia 11, 46-51; d'ailleurs, Sannazar avait utilisé la même image dans la première églogue de l'Arcadia, mais plus brièvement: «Progne ritorna a noi per tanto spazio / con la sorella sua dolce cecropia / a lamentarsi de l'antico strazio " $(1,22-24)$.

26. Après la bataille de Pharsale, dans laquelle il avait été écrasé par César, Pompée avait cherché à se réfugier en Egypte auprès de son protégé Ptolémée XIII. Mais celui-ci, craignant les représailles de César, le fit assassiner.

27. Ce même tercet sera par la suite ultérieurement exploité par Dolce. Dans la partie consacrée au défilé des personnages célèbres, il fera en effet l'objet d'une libre réécriture qui se présente de la 
Encore plus intéressant, pour le travail de «collage-réécriture " de différentes sources, se révèle le passage consacré à la reine de Palmyre Zénobie:

Et [regarde] Zenobia ch'al fin rimase vinta

Armata, et con il cor saggio et pudico.

Ell'era armata et a battaglia accinta

Et mirando i Roman, quasi smarita

Pareva, e in fronte di vergogna tinta.

(Il sogno di Parnaso, v. 350-354)

Le modèle de départ pour ces vers est, une fois de plus, le Triumphus Fame où Zénobie était présentée de la manière suivante:

[...] $\mathrm{E}$ vidi in quella tresca

Zenobia, del suo onore assai più scarsa:

bella era, e nell'età fiorita e fresca,

quanto in più gioventute $\mathrm{e}$ 'n più bellezza

tanto par ch'onestà sua laude accresca.

Nel cor femineo fu sì gran fermezza,

che col bel viso e coll'armata coma

fece temer chi per natura sprezza:

io parlo de l'imperio alto di Roma,

che con arme assalìo, ben ch'a l'estremo

fusse al nostro triumfo ricca soma.

(Triumphus Fame II, 107-117)

Tout en privilégiant la concision, Dolce retrace comme Pétrarque le portrait de cette femme réputée pour ses qualités guerrières ${ }^{28}$. Pourtant, on remarque immédiatement que Lodovico développe plutôt librement les tercets consacrés à Zénobie dans le Triumphus Fame. Pétrarque avait centré son hommage à la reine de Palmyre sur sa proverbiale chasteté, sa beauté et sa valeur guerrière qui l'avait rendue, aux yeux des Romains, redoutable en bataille ${ }^{29}$. Il avait par contre accordé moins d'espace à l'épi-

manière suivante: «e in vestir bruno / ecco Pompeo, che 'l traditor d'Egitto [Ptolémée XIII] / occise, iniquo et di pietà diggiuno " (Il sogno di Parnaso, v. 281-282).

28. Grâce à son habileté dans l'exercice des armes, en 269 av. J.-C., Zénobie avait en effet pu priver les Romains de l'Egypte et de l'Asie Mineure.

29. Sur la chasteté de Zénobie voir Historia Augusta XXIV (Tyranni Triginta), XXX, 12: "Cuius ea castitas fuisse dicitur, ut ne virum suum quidem scierit nisi temptandis conceptionibus. Nam cum semel concubisset, expectatis menstruis continebat se, si praegnans esset, sin minus, iterum potestatem quaerendis liberis dabat" (Histoire Auguste. Les empereurs romains des II et IIT siècles, édition bilingue latin-français, traduction par A. Chastagnol, Paris, Robert Laffont, 1994, p. 908) ; et Petrarca, Fam. XXI, 8, 14, 91-95: "Regnavit apud Egiptios Cleopatra, Zenobia apud Persas, que se reginam diceret Orientis, mulier fidutie ingentis clarissimeque virtutis, inter cetera - quod Cleopatre defuit - castitatis eximie, cuius laudem forme raritas geminabat " (cité d'après Francesco Petrarca, Le familiari, ed. V. Rossi-U. Bosco, vol. IV, Florence, Sansoni, 
logue de l'histoire de cette héroïne qui finit par être capturée par ses rivaux. Dans Il sogno di Parnaso ce schéma est sensiblement modifié. Dolce conserve uniquement l'évocation de la chasteté de Zénobie (mentionnée toutefois très brièvement) et sacrifie toute allusion à la beauté de cette reine et au fait qu'elle représentait pour les Romains une ennemie redoutable; il insiste en revanche sur l'image de cette femme dans le rôle de guerrière toujours prête à l'action et s'attarde sur les effets provoqués en elle par le souvenir de sa capitulation, lorsque Aurélien l'amène à Rome pour le triomphe célébrant la victoire des Romains.

L'association entre le thème des armes et celui de la chasteté semble inspirée, une fois de plus, par les Triomphes. Ces deux motifs se trouvent en effet unis aussi bien dans le Triumphus Pudicitie que dans le Triumphus Mortis. Mais c'est notamment l'incipit du premier chapitre du Triumphus Mortis qui a servi d'ébauche à Dolce comme le montre, entre autres, la définition de la fermeté morale de Zénobie, très proche de celle utilisée par Pétrarque pour Laure:

Quella leggiadra e glorïosa donna [Laure],

[...]

tornava con onor da la sua guerra,

allegra, avendo vinto il gran nemico [Amour]

che con suo 'ingengi tutto 'l mondo atterra,

non con altre arme che col cor pudico ${ }^{30}$.

Certes, les deux scènes proposées respectivement par Dolce et par Pétrarque sont diamétralement opposées à bien des égards. Tout d'abord, la «leggiadra e glorïosa donna» du Triumphus Mortis n'est qu'une guerrière symbolique qu'on n'imaginerait às aucun moment habillée comme une guerrière classique, contrairement à Zénobie qu'on se figure immédiatement dotée d'un casque, d'une cuirasse et d'un bouclier. En outre, la première est saisie en train de savourer la victoire qu'elle a remportée dans

1968). Quant à la beauté de Zénobie, voir Historia Augusta XXIV, XXX, 15 : «Fuit vultu subaquilo, fusci coloris, oculis supra modum vigentibus nigris, spiritus divini, venustatis incredibilis. Tantus candor in dentibus, ut margaritas eam plerique putarent habere, non dentes»; et Petrarca, De remediis utriusque fortune II, 94, 35: «At Zeonobia regina Orientis, inter caeteras formae laudes, tanto dentium nitore describitur, ut inter loquendum ridendumque, non dentibus sed candidis margaritis plenum os habere videretur" (cité d'après PÉTRARQUE, Les remèdes aux deux fortunes. De remediis utriusque fortune, texte établi et traduit par C. Carraud, Grenoble, Jérôme Million, 2002).

30. Triumphus Mortis I, 1-7. Ce souvenir s'est fort probablement croisé avec celui d'un passage de la chanson Amor, se vuo' ch'i' torni al giogo anticho: "[Amour] Il mio amato tesoro [Laure] in terra trova, / Che m'è nascosto, ond'io son sì mendico, / E'l cor saggio pudico, / ove suol albergar la vita mia» (Rvf270, 5-8). 


\section{UN EXEMPLE DE RÉÉCRITURE PÉTRARQUISTE}

une guerre menée contre un ennemi incorporel, Amour. La reine de Palmyre, en revanche, est immortalisée à l'occasion de sa défaite infligée par une armée entière d'ennemis en chair et en os, les Romains. Quant à la fermeté morale, chez Pétrarque elle représente l'arme qui a permis à Laure de vaincre Amour. Pour la Zénobie de Dolce, en revanche, elle n'est qu'un trait de son caractère qui s'accompagne de la capacité qu'a cette femme de savoir manier parfaitement les armes à l'instar d'un homme.

Par ailleurs, l'image de Zénobie chaste et toujours prête à livrer bataille n'est pas sans rappeler les hérö̈nes chevaleresques de Boiardo et de l'Arioste. Elle fait notamment penser à Marphise, toujours habillée comme une guerrière et désireuse de se mesurer par les armes avec les hommes. Mais l'air troublé que Dolce confere par la suite à la reine de Palmyre en la décrivant comme "quasi smarrita» (formule littéralement extraite de Pétrarque ${ }^{31}$ ), rappelle Isabelle, "in viso pallida e smarrita ", et Rodomont, "attonito e smarrito", du poème de l'Arioste. "Smarrita / o " traduit dans le Roland furieux, comme chez Dolce, un sentiment de détresse et de dépaysement, même si les raisons qui provoquent cette réaction sont différentes. Isabelle est désorientée à cause de la perte de Zerbin qui vient de mourir ${ }^{32}$; Rodomont est déconcerté face à l'aveu public et inattendu de Doralice (la femme aimée et de laquelle il croyait être aimé en retour) qui affirme lui préférer un autre ${ }^{33}$. Zénobie, quant à elle, est déroutée car elle se retrouve en situation d'impuissance et d'infériorité face au peuple ennemi duquel elle avait autrefois triomphé. A l'attitude désemparée de Zénobie, Dolce ajoute également un sentiment de honte pour la défaite subie lequel se traduit par l'expression «in fronte di vergogna tinta». Le passage concernant Rodomont semble en être encore une

31. Cf. $R v f 7,3$ et 47,6 .

32. Ludovico ARIOSTO, Orlando Furioso XXVIII, 95-97: "Standovi un giorno il Saracin [Rodomont] pensoso / [...] / vide venir per mezzo un prato erboso, / [...] / una donzella di viso amoroso / in compagnia d'un moncao barbato; / e si traeano dietro un gran destriero / sotto una soma coperta di nero. // Chi la donzella, chi 'l monaco sia, / chi portin seco, vi debbe esser chiaro. / Conoscere Issabella si dovria, / che 'l corpo [le cadavre] avea del suo Zerbino caro. / [...] // Come ch'in viso pallida e smarrita / sia_la donzella, et abbia i crini inconti; / e facciano i sospir continua uscita / del petto acceso, e gli occhi sien duo fonti / et altri testimoni d'una vita / misera e grave in lei si veggan proni; / tanto però di bello anco le avanza, / che con le Grazie Amor vi può aver stanza" (toutes nos citations d'après l'édition par les soins de S. Debenedetti et C. Segre, Bologne, Commissione per i testi di lingua, 1960).

33. «Poi lor convenzion ratificaro / in man del re [Agramant] quei duo prochi famosi [Rodomont et Mandricard], / et indi alla donzella [Doralice] se n'andaro. / Et ella abbassò gli occhi vergognosi, / e disse che più il Tartaro [Mandricard] avea caro : / di che tutti restâr meravigliosi; / Rodomonte sì attonito e smarrito, / che di levar non era il viso ardito" (Orlando Furioso XXVII, 107). 
fois source d'inspiration. Nous ne sommes pas loin en effet de «quella vergogna che gli avea [à Rodomont] la faccia tinta» devant le choix de Doralice (Orlando Furioso XXVII, 108).

Le passage concernant Zénobie n'est pas le seul à montrer l'influence $\mathrm{du}$ Roland furieux dans Il sogno di Parnaso. Nous pensons, entre autres, à la présentation des héros romains et grecs pour laquelle des souvenirs du Roland Furieux viennent entourer la réécriture d'un célèbre passage de Pétrarque:
Così intramo per certe horrende porte
Tutte d'acciaro, et pervenimo in parte
Dove surgea un Castel potente et forte.
"Hor vedi quivi il buon popol di Marte,
Che meritò di Lauro ornar i crini
Sol colla spada, et non per scriver carte;
Et in disparte vedi i perregrini».
Così disse la Donna. Indi d'alcuno
M'apperse il nome, e i gesti alti et divini.
(Il sogno di Parnaso, v. 271-279)

Les portes d'acier qui s'ouvrent sur le château abritant les généraux romains et leurs ennemis, se présentent en effet comme une variante du château en acier construit par le magicien Atlante, que l'Arioste avait à son tour repris de Boiardo ${ }^{34}$. Le guide qui dévoile un par un les noms et les hauts faits des héros romains et de leurs rivaux évoque en revanche la magicienne Mélisse en train de révéler à Bradamante, dans la grotte de Merlin, l'identité des futurs descendants de cette dernière et les actions dont ils seront protagonistes (Orlando Furioso III, 1-62). Afin de souligner la ressemblance entre le guide du Sogno di Parnaso et Mélisse, Dolce reprend, à la fin du passage, presque à la lettre les mots que Mélisse adresse à Bradamante avant d'évoquer les esprits de ses héritiers: "Se i nomi et $\mathrm{i}$ gesti di ciascun [de tes descendants] vo' dirti» (Orlando furioso III, 23). Parmi les légères modifications que Dolce a apportées à la phrase de l'Arioste, on remarque immédiatement le passage de la forme active à la forme passive. Dans Il sogno di Parnaso, c'est en effet le protagoniste-narrateur qui nous informe que le nom et l'identité de chaque personnage lui

34. Cf. ARIosto, Orlando Furioso II, 41-43; III, 63; IV, 7 et XII, 21; et Matteo Maria BOIARDO, Orlando Innamorato II, III, 28 et II, XVI, 17. Au souvenir de ces passages, où revient toujours l'image de l'acier, se mélange également celui du palais en acier du dieu Mars décrit par Boccace dans son Teseida: «in questa [forêt] vidde la ca'dello dio / armipotente [Mars], questa edificata / tutta d'acciaio splendido e pulio, / [...] e le porte eran d'etterno adamante" (Giovanni BocCACCIO, Teseida, VII, 32, ed. A. Limentani, in Tutte le opere..., cit., vol. II, 1964). 
ont été révélés par le guide, alors que chez l'Arioste c'est Mélisse ellemême qui annonce d'être chargée de la même tâche à l'égard de Bradamante à qui elle sert de guide. Afin de laisser le vers de l'Arioste facilement reconnaissable, Dolce a également légèrement retouché la tournure de la phrase et a remplacé le pronom "ciascuno" et le verbe "dire" par des synonymes. Il a en revanche laissé pratiquement intact le syntagme «i nomi e i gesti» auquel il a ajouté les adjectifs «alti» et «divini» qui constituent une autre référence à l'Arioste ${ }^{35}$.

Au milieu de ces réminiscences de l'Arioste, Dolce emboîte donc le discours direct qui se ressent de l'influence d'un passage de la chanson de Pétrarque $O$ aspectata in ciel beata e bella dédiée à Giacomo Colonna:

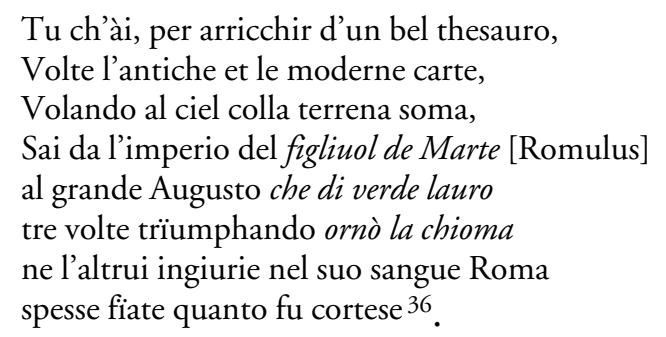

Dolce réemploie certains mots qu'il adapte à ce nouveau contexte. Ainsi, il renverse le sens du deuxième vers. L'objet de la célébration n'est plus une seule personne, mais un groupe et la raison de l'hommage ne réside pas dans les qualités intellectuelles, mais dans des valeurs guerrières. Dolce utilise également l'image du couronnement qu'il réécrit à sa façon. Par ailleurs, il remplace la périphrase faisant référence à Romulus par une autre, désignant les héros romains, empruntée au Triumphus Fame ${ }^{37}$. Pour présenter les ennemis des romains, en revanche, Lodovico s'appuie sur deux Triomphes. Le vers "et in disparte vedi i perregrini» (Il sogno di Parnaso, v. 277) est en effet conçu en pensant d'une part à l'expression que Pétrarque avait utilisée dans son Triumphus Eternitatis pour démarquer

35. «I gesti alti et divini» des héros du monde grec et latin que le guide de Dolce s'apprête à raconter rappellent en effet le célèbre vers de l'exorde du Roland furieux «l'alto valore e'chiari gesti » que l'Arioste s'engage à raconter au sujet de Roger (I, 4), ainsi que «li alti gesti», toujours accomplis par Roger, de la première des Satires du même auteur (v. 139-141; in Opere minori, ed. C. Segre, Milan-Naples, Ricciardi, 1954).

36. Rvf 28, 76-83. Ce passage avait déjà été précédemment exploité pour présenter Platon. Voir supra, p. 7.

37. "Pien d'infinita e nobil meraviglia, / presa a mirar il buon popol di Marte» (Triumphus Fame II, 1-2). La périphrase "popol di Marte» se trouve aussi dans la chanson Spirto gentil, che quelle membra reggi $($ Rvf 53, 26). 
des damnés ceux pour qui la seule richesse était celle intérieure ${ }^{38}$, d'autre part aux «pellegrini egregi» du Triumphus Fame (II, 7) qui désignaient déjà les adversaires des Romains.

Le recours à l'Arioste en dehors des parties concernant le défilé des personnages ne figure pas comme un cas isolé. Libre des schémas imposés par une description des personnages historiquement établie, Dolce peut bâtir son texte en s'appuyant, en plus de Pétrarque, sur un certain nombre d'autres auteurs. Ainsi, la première partie du vers "vogli in qua gli occhi, et mira un'altra setta" (Il sogno di Parnaso, v. 253), qui marque la transition d'un groupe de personnages à un autre, se présente comme un véritable calque de "vogli in qua gli occhi e mira in su quel corilo» de l'Arcadie $(12,22)$. De même, le vers "nell'alma intrò con le parole il suono" (Il sogno di Parnaso, v. 136), est le résultat d'une légère retouche apportée au vers "ne l'alma dentro le parole e s'l suono", contenu dans la première des trois chansons que Lavinello déclame à la reine dans les Asolani de Bembo $^{39}$. La réécriture peut également aller au-delà de la citation d'un simple vers et intéresser, par exemple, un tercet entier; dans ce cas il peut s'agir de la réécriture d'après un seul auteur ou d'un mélange de souvenirs de plusieurs auteurs. Voici le moment où le guide dévoile à Lodovico que la montagne à l'aspect si effrayant qui s'est dressée brusquement devant lui est très convoitée par tout individu en quête de gloire, car sur son sommet se trouve le siège des Muses: "Ivi [sur le sommet du Parnasse] sta la celeste et sacra Prole / Del Sommo Giove, et ivi è 1 fonte santo, / In cui si specchia et sì vagheggia il Sole» (Il sogno di Parnaso, v. 43-45). La première moitié du tercet contient la réminiscence d'un vers de Sannazar du célèbre sonnet Eran le Muse intorno al cantar mio ("per voi seme gentil del sommo Giove $\left.^{40} »\right)$ à laquelle Dolce ajoute le souvenir des adjectifs combinés "celeste et sacra» repris de Tebaldeo et Niccolò da Correggio ${ }^{41}$. La

38. «e 'n disparte, color che sotto 'l freno / di modesta fortuna ebbero in uso / [...] di godersi in seno" (Triumphus Eternitatis 118-120). Lodovico, en revanche, la réemploie pour séparer les romains de leurs adversaires.

39. Voir Pietro Bembo, Asolani, ed. G. Dilemmi, Florence, Accademia della Crusca, 1991, livre III, chapitre 8. Dolce avait d'ailleurs déjà utilisé ce passage de Bembo pour la rime harai / terrai / mai des vers 74-78.

40. Sonetti e canzoni 2, 9; l'écho de Sannazar reviendra aussi dans le tercet «Quelle, che con giocondo e lieto aspetto / Gli [à Apollon] stanno intorno, son l'alme sorelle / Di Giove, seme altero et benedetto" (Il sogno di Parnaso, v. 196-198).

41. «Lasso, quando serà ch'io satisfaccia / piangendo a quei celesti e sacri lumi / che al mio partir si fêr dui fiumi" (Tebaldeo, Rime 159, 1-2); "Placidi aspecti di benigne stelle, / celesti, sacri, armonici concenti / aer, foco, acqua, terra, alti elementi» (NICCOLO DA CORREGGIO, Rime 233, 1 3, in IDEM, Opere, ed. Antonia Tissoni Benvenuti, Bari, Laterza, 1969). 
deuxième partie du tercet est en revanche inspirée d'un passage de la chanson Stagione aprica, natural tesauro de Niccolò da Correggio dans lequel une "villanella cerca il chiaro fonte / e in quel si lava e lì tutta si specchia" (Rime 361, 89-90). Dans Il sogno di Parnaso, «il chiaro fonte» est devenu "'l fonte santo" (souvenir du Teseida de Boccace ${ }^{42}$ ), où «si specchia» non pas une jeune femme, mais le soleil qui regarde avec complaisance l'image que la source renvoie de lui. Ce soleil qui s'abandonne à cet acte de vanité, exprimé par le verbe "si vagheggia", rappelle en revanche notamment l'Aurore qui, dans l'incipit d'un sonnet toujours de Correggio, elle aussi "si vagheggia", fière de sa beauté: "Quando di rose coronata e adorna / Al balcon d'oriente si vagheggia / La bella Aurora » (Rime extrav.38, 1-3).

Une attention particulière doit être accordée au passage où Dolce, après un long parcours qui s'est déroulé dans un paysage caractérisé par l'absence de toute forme de vie, parvient avec son guide dans un lieu animé par des gens qui suscitent sa curiosité, à laquelle le guide répond de la manière suivante:

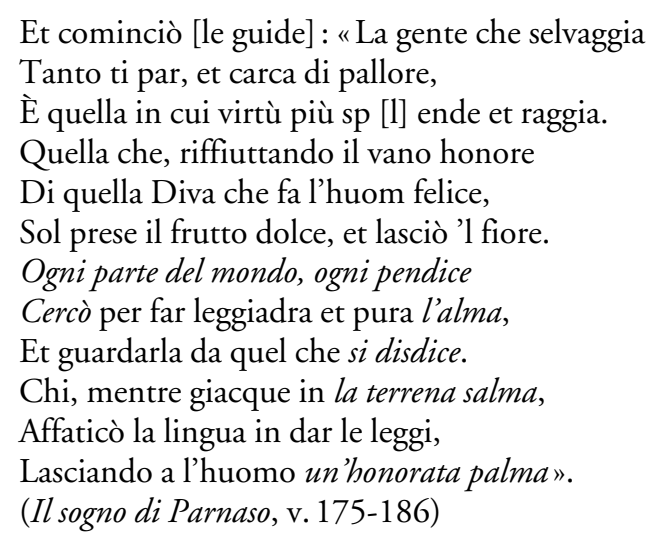

Le premier tercet mélange des souvenirs tirés de plusieurs auteurs. La pâleur de la "gente selvaggia» (réminiscence de Cino ${ }^{43}$ ) renvoie à la fois à la description que Dante fait des gourmands dans son Purgatoire, à celle de la famine dans les Métamorphoses d'Ovide, ainsi qu'à celle des Harpies dans le Roland furieux ${ }^{44}$. Mais Dolce renverse complètement le sens de ces

42. "Così gli abbiam [les vers de Boccace], rorati al fonte santo, / licenziati a gire in ogni canto" (Teseida, Risposta delle Muse 15-16).

43. Cf. Cino da Pistoin, Rime 43, 2 et 103, 1 et 6 (in Mario Marti, Poeti del Dolce stil nuovo, Florence, Le Monnier, 1969).

44. "Nelli occhi era ciascuna oscura e cava, / palida nella facci, e tanto scema, / che dall'ossa la pella s'informava» (Purgatorio XXIII, 22-24); «cava lumina, pallor in ore [...] / Ossa sub incurvis 
trois représentations pour l'adapter à ses fins. Ainsi, la pâleur qui, dans les descriptions de Dante, Ovide et l'Arioste, concourt à susciter un sentiment de répugnance, engendre, dans Il sogno di Parnaso, un regard de déférence. De même, les adjectifs "pallida et smorta» du vers 168, que Dolce emprunte au passage de l'Arioste concernant les monstres mythologiques, ne véhiculent plus un sentiment de laideur comme c'est le cas dans le Roland furieux, mais ils évoquent la grande dignité morale qui caractérise les personnages.

Quant à l'image de la vertu qui rayonne de ces gens, elle semblerait suggérée par le célèbre passage du Purgatoire (qui suit d'ailleurs l'épisode des gourmands) où Stace explique à Dante que, dès que l'âme arrive dans le lieu du châtiment éternel ou de la pénitence purificatrice auquel elle a été assignée, elle est entourée par un espace aérien sur lequel commence immédiatement à opérer la vertu "formativa» qui se trouve en elle: "Tosto che loco lì la [l'âme] circunscrive, / la virtù formativa raggia intorno/ così e quanto nelle membra vive» (Purgatorio, XXV, 88-90).

A la différence de ce premier tercet, qui se présente riche de suggestions provenant de différents auteurs, les trois autres tercets résultent d'un travail de réécriture très libre menée sur un passage de la chanson de Sannazar In la morte di Pier Leone auquel Dolce semble presque répondre, comme on le faisait dans les échanges de sonnets:

O padre, o signor mio, l'uscir fora, come tu sai, non è permesso a l'alma, né far si dee, se 'l ciel non vòle ancora; ché 'l dispregiar de la terrena salma a quei con più vergogna si disdice, che più braman d'onor aver la palma.

- Ogni riva del mondo, ogni pendice cercai - rispose [Pier Leone] - e femmi un altro Ulisse filosofia, che suol far l'uom felice.

(Sonetti e canzoni 101, 58-66)

Dolce emprunte à Sannazar les mêmes rimes auxquelles il donne toutefois une nouvelle disposition. Il garde intacte la rime alma / salma / palma, mais il la déplace après la rime felice / pendice / disdice à laquelle en revanche il fait subir une inversion du premier et du dernier terme. Mais Dolce ne se limite pas à modifier la position des rimes. Il adapte aussi ces 
dernières à un nouveau contexte. Du reproche que Sannazar adresse à Pier Leone pour s'être ôté la vie, action qu'il considère comme encore plus méprisable lorsqu'elle est commise par ceux qui sont à la recherche d'honneur, on passe à l'exaltation, par Dolce, de tous ceux qui méritent d'être honorés pour avoir choisi une vie basée non pas sur les apparences, mais sur des valeurs essentielles. Pour écrire cette louange, Dolce se sert encore du même passage de Sannazar. Il commence en exploitant le dernier des trois tercets dont il remploie le premier et le dernier vers qu'il insère dans deux tercets différents. Le dernier vers de Sannazar, légèrement retouché, se retrouve ainsi à faire partie du premier tercet de Dolce où il finit par véhiculer une valeur négative. La "Diva» qui fait le bonheur des hommes ne semblerait être en effet rien d'autre que la richesse matérielle. La philosophie, qui dans le passage de Sannazar représente un bien précieux car elle nourrit et enrichit l'esprit, est donc remplacée, chez Dolce, par un bien éphémère qui attire les hommes superficiels, lesquels n'accordent de l'importance qu'aux apparences. Toutefois, l'opinion négative exprimée par Dolce à l'égard de la richesse est glissée à l'intérieur d'un tercet qui a une finalité positive dans la mesure où il exalte ceux qui ont fui tout ce qui est futile pour se fixer des buts plus nobles, comme l'indique la métaphore qui oppose la fleur, qui n'est que pure beauté extérieure, au fruit, symbole de la substance qui manque à cette dernière.

Dolce revient d'ailleurs sur ce même concept dans les deux tercets suivants où il insiste sur la volonté constante de ces hommes de s'éloigner des passions mondaines et d'accomplir des actes méritoires qui leur ont valu la palme de l'honneur. Encore une fois, Dolce recourt à Sannazar pour exprimer cette idée. Ainsi, pour mieux faire ressortir l'image des hommes qui cherchent partout le chemin de la vertu, Dolce reprend la phrase "Ogni riva del mondo, ogni pendice cercai» en remplaçant "riva" par "parte» et en passant de la première à la troisième personne du verbe "chercher» imposée par le discours narratif. La conclusion de la louange s'articule en revanche autour des expressions «terrena salma» et "honorata palma» que Dolce emprunte au deuxième tercet de Sannazar. Mais une fois de plus, ces deux expressions sont destinées à être insérées dans un autre contexte afin de produire un effet diamétralement opposé à celui recherché par Sannazar. Ce dernier avait centré ses vers sur la désapprobation que méritent tous ceux qui méprisent la «terrena salma», c'est à dire la vie. Dolce, par contre, remplace la réprobation par un éloge dont font l'objet tous ceux qui, contrairement aux premiers, ont été capables de vivre intensément leur vie en s'efforçant d'être utiles aux autres. De même, le profond désir «d'onor aver la palma», resté irréalisé chez ceux 
qui mettent fin à leurs jours et qui sont pour cela réprimandés par Sannazar, trouve en revanche sa concrétisation chez les destinataires de la louange de Dolce auxquels ce dernier confere "un'honorata palma" pour la vertu dont ils ont fait preuve au cours de leur vie.

La réécriture que Dolce propose de ce passage de Sannazar reste pourtant exceptionnelle. Dolce reprend en effet rarement plus d'une rime d'un même passage, car cela impliquerait des contraintes évidentes dans la rédaction d'un nouveau texte qui souvent n'a rien en commun avec celui qui fournit les rimes. Comme nous l'avons vu, sa démarche préférée consiste à mélanger dans un même vers ou dans un tercet des suggestions provenant de Pétrarque avec celles d'un autre ou de plusieurs autres auteurs ${ }^{45}$.

En passant d'une réécriture à l'autre, Dolce nous fait ainsi parvenir à la fin de son voyage. Le guide invite alors le protagoniste à redescendre la montagne et à rejoindre le commun des mortels:

«Hor contento tu, Amico, gli occhi abbassa,

Che più veder' non lice et qui ti lasso.

Per te puoi rieder tra la gente bassa.

Facil cosa ti sia ritrar il passo

Ch'al discender è dritta et piana via,

Al poggiar aspra, et rende l'huomo lasso.»

Intanto il sonno et ella fuggì via.

(Il sogno di Parnaso, v. 457-463)

C'est sur ces mots que Dolce conclut son ouvrage, en parfait accord avec la structure portante du poème, où la Comédie joue un rôle fondamental

45. Voir, par exemple, les vers que Dolce met dans la bouche du guide pour insister sur le caractère exceptionnel de l'aventure que le destin lui a réservée: "Qual gratia, o qual destino il ciel ti diede, / Ch'a tant'alto camin t'impenna l'ale?» (Il sogno di Parnaso, v. 35-36). Incontestable source d'inspiration pour ces vers est le dernier tercet du sonnet de Pétrarque Io son sì stanco sotto 'l fascio antico: "Qual gratia, qual amore, o qual destino / Mi darà penne in guisa di colomba / Ch'i' mi riposi, et levimi da terra?» ( $R v f 81,12-14)$. Dolce reprend presque intégralement le premier vers de Pétrarque en supprimant seulement l'élément de l'amour qui ne s'adapte pas à son contexte. Ensuite, il réécrit les deux autres vers en gardant l'image de l'homme qui aspire à posséder des ailes pour pouvoir accéder à des endroits inaccessibles aux mortels. Pour Pétrarque, il s'agissait de s'élever jusqu'à Dieu, pour Dolce jusqu'à Apollon et à son royaume, symbole de la renommée, désigné par l'expression «alto camin" qui semblerait suggérée par Tansillo (Canzoniere 23, 68-69, ed. E. Pèrcopo , T.R. Toscano, Naples, Liguori, 1996). Quant à l'expression «t'impenna l'ale» (inspirée des "penne in guisa di colomba» qui apparaissent dans le vers de Pétrarque), on peut en revanche remonter aux Rime de Boccace: «In quel [sc. visage] risplende chiara bellezza / che 'l ciel adorna e che n'impenna l'ali / a l'alto vol con penne di virtute» (Giovanni BocCACCIO, Rime, Parte prima, XVI, 12-14, in Tutte le opere..., cit., vol., V, 1, 1992). 
dans le récit de l'ascension du Parnasse. La façon dont le guide prend congé de Lodovico en le rassurant quant à la facilité de refaire à l'envers le chemin parcouru jusqu'à là, est en effet inspirée d'un autre passage du poème de Dante où Virgile répond aux âmes qui viennent de débarquer sur la plage du Purgatoire et qui cherchent de l'aide pour trouver le chemin qui mène à la porte de celui-ci :

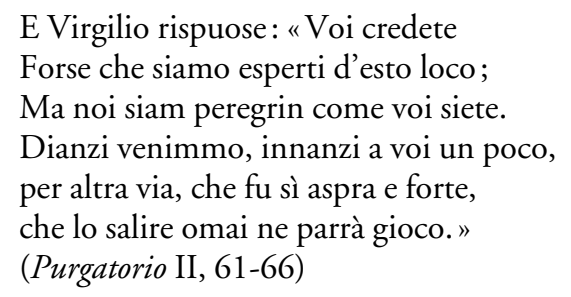

À l'instar du guide de Dante qui oppose le parcours difficile de l'Enfer à celui du Purgatoire, plus aisé, le guide de Lodovico insiste sur l'opposition entre la difficulté de gravir le Parnasse et l'extrême facilité avec laquelle, en revanche, on le descend. La ressemblance entre les discours tenus par les deux guides passe aussi par le lexique. La "via» qui apparaît "aspra» lorsqu'on gravit le Parnasse rappelle en effet la "via [...] aspra et forte» que Dante a parcourue dans l'Enfer. Toutefois, pour souligner le contraste entre le parcours qui mène au siège des Muses et celui du retour qui reconduit à la vie de tous les jours, Dolce choisit de recourir à Pétrarque qui lui suggère autant l'idée de la «via dritta» et de la "via piana» que l'emploi antithétique des adjectifs «aspro" et "piano ${ }^{46}$ ». Dans le sonnet dédié à Sennuccio del Bene, Pétrarque avait en effet déjà placé ces adjectifs l'un à côté de l'autre afin de faire ressortir le comportement antinomique de Laure: "Qui tutta humile, et qui la vidi altera, / or aspra, or piana, or dispietata, or pia» $(R v f 112,5-6){ }^{47}$.

Pour souligner l'issue de son rêve et le retour à la réalité, Dolce retourne, en revanche, à Dante, et plus précisément au célèbre début du chant neuf du Purgatoire: Dante y passe, pendant son sommeil, de

46. Quant à l'emploi de "via dritta», cf. Rvf 91,7 (" e seguir lei per via dritta expedita»), 119, 84 (" e se mai de la via dritta mi torsi») et 261, 7-8 (" qual è dritta via / di gir al ciel»); en ce qui concerne "via piana», cf. $R v f 105,70-71$ («i segni del bel volto / che mi conducon per più piana via / a la speranza mia») et 244, 1-2 (« Il mal mi preme, et mi spaventa il peggio, / al qual veggio sì larga et piana via").

47. Toutefois, pour ce passage Dolce est également tributaire de l'Arioste. Cf. Orlando furioso XXII, 46 qui inspire en effet à Lodovico Dolce non seulement l'idée des deux chemins qui présentent des difficultés diamétralement opposées, mai aussi les adjectifs «dritta et piana» en référence au mot "via". 
l'Antipurgatoire au Purgatoire proprement dit à l'aide d'une femme nommée Lucia, comme il l'apprendra à son réveil par Virgile qui a assisté à toute la scène. Des échos de cet épisode se trouvent déjà dans l'exorde du Sogno di Parnaso. Dolce en avait repris de nombreux éléments, liés au moment où Dante s'endort et rêve, pour les adapter au début de son propre rêve ${ }^{48}$. Au terme de ce rêve, Dolce s'inspire du vers par lequel se conclut l'épisode dantesque qui lui a donné l'idée de faire du Parnasse un Purgatoire pour les écrivains désireux d'être admis au royaume d'Apollon, siège de la gloire poétique: "poi ella [Lucie] e 'l sonno ad una se n'andaro" (Purgatorio IX, 63). Pour Dolce qui vient de voir le Paradis destiné aux poètes, il est désormais temps de sortir de la fiction. Toutefois, la formule qu'il utilise à cette fin, porte également les traces évidentes de celle que Pétrarque, en s'inspirant à son tour de Dante, emploie pour conclure la chanson Quando il soave mio fido conforto: "et dopo questo si parte ella e'l sonno» (Rvf359, 71) ${ }^{49}$.

Par le recours à cette locution, commune à Dante et à Pétrarque, et dont une autre variante est fournie par Bembo dans l'un des sonnets qui $s^{\prime}$ inscrivent dans le genre du "rêve consolateur " ${ }^{50}$, Lodovico Dolce pouvait atteindre deux buts. D'une part, celui de terminer son ouvrage en parfaite symétrie avec son exorde, où des échos de Dante et de Pétrarque, les deux sources qui dominent $I l$ sogno di Parnaso, viennent là aussi se confondre. D'autre part, celui de rendre un dernier hommage à Pietro Bembo, figure fortement représentative du pétrarquisme dont la célébration constitue la raison de cet ouvrage qui se présente comme la déclaration, par Dolce, de son adhésion à ce courant poétique.

48. Voir ici p. 59 et note 22.

49. La même image avait été également utilisée par Pétrarque dans la première rédaction du Triumphus Fame: "e 'l sonno, e quella ch'anchor apre e serra / il mio cor lasso, a pena eran partiti, / ch'io vidi incominciar un'altra guerra" (Triumphus Fame Ia, 10-12).

50. «Giosene appresso il sonno, et ella, insieme / co' miei diletti e con la notte intorno" (Pietro Bembo, Rime, in IDEm, Prose e Rime..., cit., 90, 12-13). 